\title{
Flüssiger Schwefelphosphor;
}

\author{
von
}

\section{Hans Sohulze.}

Trotz zahlreicher Arbeiten über die flüssigen Schwefelphosphorverbindungen ist deren chemische Constitution noch immer nicht endgiltig aufgeklärt. Die eingehendsten Untersuchungen über diese Körper verdanken wir Berzelius. Derselbe unterwarf in einer ausgezeichneten Arbeit ${ }^{1}$ ), der letzten des grossen Chemikers, das Verhalten zwischen Phosphor und Schwefel sorgfältiger Untersuchung und gelangte auch in Bezug auf die längst bekannten, durch Zusammenfliessen von Phosphor und Schwefel bei wenig erhöhter Temperatur entstehenden Körper zu bemerkenswerthen Resultaten, die noch heute für maassgebend gelten und in allen chemischen Lehrund Handbüchern zu finden sind. Berzelius fand nämlich, dass jene Elemente zwei flüssige Verbindungen, das Phosphorsulfuret $\mathrm{P}_{4} \mathrm{~S}$ und das unterphosphorige Sulfid $\mathrm{P}_{2} \mathrm{~S}$, zu bilden vermögen, welche beide in einer $W$ asserstoffatmosphäre unverändert destillirbar sind, und von denen ersteres einige Grade unter Null, letzteres in einem Gemisch von Kochsalz und Schnee erstarrt. Aus einem flüssigen Sulfide, zu dessen Bereitung mehr Phosphor verwendet wird, als der Formel $\mathrm{P}_{4} \mathrm{~S}$ entspricht, krystallisirt der überschüssige Phosphor aus, während aus Auflösungen des Schwefels in unterphosphorigem Sulfid schöne Krystalle der Verbindung $\mathrm{P}_{2} \mathrm{~S}_{12}$ (nach Dupré $\left.{ }^{2}\right) P_{2} S_{6}$, nach Faraday ${ }^{3}$ ) aber Schwefelkrystalle) anschiessen. Sprechen nun einerseits diese Angaben dafür, dass wir in den flüssigen Phosphorsulfiden wirkliche chemische Verbindungen vor uns haben, so erwecken andererseits zwei in Gmelin-Kraut's Handbuche ${ }^{4}$ ) befindliche Notizen, nach deren Autoren ich vergebens die chemische Literatur durch-

1) Ann. Chem. Pharm. 46, 129.

3) Dies. Journ. 21, 243.

3) Ann. chim. phys. 7, 71.

4) Gmelin-Kraut 1 [2], 236 u. 237.

Journal f. prakt. Chemie [2] Bd. 22. 


\section{Schulze: Flüssiger Sehwefelphosphor.}

späht habe, ernste Zweifel an der chemischen Individualität dieser Körper. Die eine der Angaben besagt, dass die beim Zusammenbringen von Schwefel und Phosphor unter $100^{\circ}$ entstehenden Verbindungen sich verschiedenen Lösungsmitteln (und chemischen Einwirkungen) gegenüber wie Gemische von Schwefel und Phosphor verhalten, indem ihnen vorzugsweise der eine Bestandtheil entzogen werde, indess der andere mehr oder weniger unverändert zurückbleibe. Die zweite Notiz bietet das einzige Detail zu dieser Behauptung; Schwefelkohlenstoff soll, wie sie angiebt, aus gleich viel Schwefelphosphor vorzüglich den Phosphor ausziehen und den meisten Schwefel in Verbindung mit etwas Phosphor abscheiden. Vor Kurzem bèkämpfte G. Ramme ${ }^{1}$ ) auf Grund anderer Thatsachen die nach Berzelius' Vorgange allgemein geltende Annahme, dass die flüssigen Phosphorsulfide wirkliche chemische Verbindungen seien; er erklärt dieselben für ölige "Gemische", weil sich beim Zusammenfliessen von Phospor und Schwefel unter warmem Wasser keine Temperaturerhöhung beobachten lasse, und weil, wenn man jene Flüssigkeiten mit Wasserdampf destillire, Phosphor übergehe und Schwefel mit geringem Phosphorgehalte zurückbleibe. Bekanntlich erleidet aber flüssiger Schwefelphosphor durch Wasserdampf Zersetzung, und wenn sich diese auch wenig energisch vollzieht, so kann doch ein auf diese Weise erlangtes Resultat nicht als exact gelten. Minder anfechtbare Resultate erzielt man, wenn man den Wasserdampf durch eine chemisch völlig unwirksame Atmosphäre, durch Wasserstoff, Kohlensäure oder Stickstoff ersetzt. Dadurch gewinnt man auch unmittelbare Fühlung mit Berzelius' Untersuchungen, der bekanntlich berichtet, dass beide flüssigen Sulfide in der Wasserstoffatmosphäre unzersetzt destillirbar seien. Bevor ich jedoch $\mathrm{zu}$ den eigenen Beobachtungen in dieser und anderer Richtung übergehe, sei einiger bemerkenswerther Thatsachen gedacht, welche bei der Darstellung des flüssigen Schwefelphosphors aus den Elementen beobachtet wurden.

1) Ber. Berl. chem. Ges. 12, 1350. 


\section{Darstellung des flüssigen Schwefelphosphors.}

Nachdem längst bekannt war, dass Phosphor und Schwefel - unter Wasser erwärmt - zu einem flüssig bleibenden Körper zusammenschmelzen, fand Wicke ${ }^{1}$ ), dass diese Vereinigung schon bei gewöhnlicher Temperatur, also ohne äussere Wärmezufuhr sich vollzieht. Er giebt an, es bilde sich hierbei immer nur die Verbindung $\mathrm{P}_{4} \mathrm{~S}$; bei Zusatz von mehr Schwefel bleibe dieser unverändert zurück oder es entstehe eine Lösung von Schwefel in $\mathrm{P}_{4} \mathrm{~S}$, aus der sich bei längerem Aufbewahren an einem kühlen Orte Schwefel in guten Krystallen absetze. Berzelius beschreibt indess auch $\mathrm{P}_{2} \mathrm{~S}$ als wohl charakterisirte chemische Verbindung, welche beim $\mathrm{Ab}$ kühlen als solche erstarre.

Auffälliger Weise wollte es mir anfangs nicht gelingen, aus wasserhellem, nach Wöhler's vortrefflicher Methode ${ }^{2}$ ) gereinigtem Phosphor und Stangenschwefel, der beim Verbrennen nur Spuren eines Rückstandes gab, einigermaassen klares Phosphorsulfid zu gewinnen. Ich verfuhr bei diesen Versuchen derart, dass ich den sorgfältig getrockneten und in Stangenform befindlichen Phosphor mit Schwefel in erbsengrossen Stücken in eine mit Kohlensäure gefüllte Glasröhre einschloss. Die Einwirkung begann sofort; die Berührungsstellen zwischen Phosphor und Schwefel wurden feucht, indess der Schwefel tief in die glatten Flächen des Phosphors einschmolz. Flüssiges Sulfid benetzte binnen Kurzem in immer reichlicheren Mengen die Wandungen der Röhre, während die Phosphorstangen durch Abschmelzung mehr und mehr verschwanden. Bei häufigem Durchschütteln vollzog sich dieser Vorgang ziemlich rasch, liess sich aber schon durch schwache Erwärmung der Röbre auf $30-40^{\circ}$ ganz ausserordentlich beschleunigen. Es bedarff kaum der Erwähnung, dass die Schnelligkeit, mit der dieses Zusammenfliessen sich vollzieht, ganz wesentlich auch vom Zertheilungsgrade, also der Grösse der wirksamen Oberfläche beider Compo-

1) Ann. Chem. Pharm. 86, 115.

2) Daselbst 45, 249. 
nenten abhängt. Ich erhielt jedoch bei zahlreichen, derweise angestellten Versuchen nie klare Präparate. Die stark getrübten Flüssigkeiten hellten sich aber auf, wenn die Röhren längere Zeit in verticaler Stellung sich befanden, während die Ursache der Trübung, ein lockeres weissgelbes Pulver, zu Boden sank. Durch aufmerksame Beobachtung des Schmelzvorganges liess sich leicht constatiren, dass diese auch beim Erwärmen ungelöst bleibenden Theilchen dem Schwefel entstammten. Weitere Versuche ergaben, dass Schwefelblumen, sowie völlig reiner, aus Sodarückständen bereiteter Stangenschwefel beträchtlich stärker getrübtes Phosphorsulfid lieferten, als das zuerst verwendete Material, dass aber aus Schwefelkohlenstoff krystallisirter Schwefel mit dem Phosphor zu klarem, durchsichtigen Oel zusammenfloss, ohne eine Spur unlöslichen Rückstandes zu hinterlassen. Dieser letzte Versuch gab den Fingerzeig, welcher zur Lösung des Räthsels führte; und zwar liegt diese Lösung darin, dass nur die in Schwefelkohlenstoff lösliche Modification des Schwefels sich mit Phosphor zu flüssigem Sulfid zu vereinigen vermag. Die einen mehr oder minder stark getriibten Schwefelphosphor liefernden Schwefelsorten enthielten neben löslichem Schwefel grössere oder geringere Mengen der unlöslichen Modification, und unlöslicher Schwefel war es, der die Trübungen verursachte. Doch ist zu bemerken, dass dieser nicht absolut unfähig zur Bildung flüssigen Schwefelphosphors ist. Bei gewöhnlicher Temperatur allerdings ist keine Spur einer Vereinigung mit Phosphor zu beobachten. Der aus Schwefelblumen dargestellte unlösliche Schwefel bestäubt die Oberfläche des trocknen, ihm dargebotenen Phosphors, ohne dass selbst nach wochenlangem Liegen ein Feuchtwerden derselben wahrzunehmen ist. Auch schmelzender Phosphor ist nicht im Stande, auf unlöslichen Schwefel einzuwirken, und erst bei ungefähr $80^{\circ}$ wird letzterer unter Bildung klaren, flüssigen Schwefelphosphors allmählich aufgelöst - ein Process, der sich bei einem Verhältniss von einem Theil Schwefel auf vier Theile Phosphor $\left(\mathrm{P}_{4} \mathrm{~S}\right.$ entsprechend) und bei $90^{\circ}$ bis $95^{\circ}$ rasch vollzieht. Der sogenanute weiche, beim Eingiessen von hoch erhitztem Schwefel in kaltes Wasser ent- 
stehende Schwefel fliesst bei gewöhnlicher Temperatur gleichfalls nicht mit Phosphor zusammen; in schmelzendem Phosphor wird er gelb und undurchsichtig und liefert bei andauerndem Erwärmen flüssiges Sulfid, geht jedoch nur zum kleinen Theile in solches über. Dieses Verhalten hat nichts Auffälliges, da amorpher Schwefel reich an der unlöslichen Modification ist. Die klaren Krystalle des wohl mit Unrecht als besondere Modification aufgefassten prismatischen Schwefels ${ }^{1}$ ) vereinigen sich ebenso leicht, wie die aus Schwefelkohlenstoff erhaltenen Octaëder mit Phosphor zu flüssigem Schwefelphosphor, und zwar bewahren sie bis zum letzten Momente des Verschwindens ihre Klarheit.

Gleichviel nun, ob wir in dem flüssigen Producte der Vereinigung von Phosphor und Schwefel eine chemische Verbindung vor uns haben oder nicht: das abweichende Verhalten des löslichen und des unlöslichen Schwefels gegen weissen Phosphor bietet in beiden Fällen eine überaus scharfe Kennzeichnung dieser beiden Modificationen, die gegenüber den gut charakterisirten Allotropien anderer Elemente, insbesondere des Phosphors und des Kohlenstoffs, bisher nicht mit gleicher Bestimmtheit als solche unterschieden wurden. Für den ersten Fall bedeutet dieses Verhalten eine wesentliche Abweichung in den chemischen Eigenschaften zweier Modificationen desselben Elementes; für den zweiten Fall ist es in Parallele zu stellen mit dem Verhalten beider Modificationen zu Schwefelkohlenstoff. Es ist selr bemerkenswerth, dass auch Phosphor nur in seiner in Schwefelkohlenstoff löslichen Modification zur Bildung flüssigen Schwefelphosphors befähigt ist, so dass sich das interessante Verhalten, welches beide Elemente bei niederen Temperaturen zu einander zeigen, in dem Schlusssatze zusammenfassen lässt: Phosphor und Schwefel vereinigen sich nur dann zu flüssigem Schwefelphosphor, wenn sie in den durch Löslichkeit in Schwefelkohlenstoff charakterisirten Modificationen mit einander in Berührung kommen.

Wie bereits erwähnt, konnte G. Ramme beim Zusam-

1) Vergl. Michaelis, Lehrb. d. anorg. Chem. 1, 542. 
menfliessen von Phosphor und Schwefel unter warmem Wasser keine Steigerung der Wärme wahrnehmen. Eine Wiederholung dieses Versuchs schien mir wünschenswerth, da sein Resultat von Bedeutung für die Frage ist, ob die Producte der Vereinigung chemische Individuen sind oder nicht. Ich verfubr in der Weise, dass ich circa $100 \mathrm{Grm}$. unter reinem Wasser granulirten weissen Phosphor mit ca. $30 \mathrm{Grm}$. gepulvertem, aus Schwefelkohlenstoff krystallisirtem Schwefel mischte; beide Substanzen besassen dieselbe Temperatur von $20^{\circ}$; sie befanden sich in einem Cylinder, der bis über den Rand in eine grössere Menge Wasser von gleichem Wärmegrade eingetaucht war. Die Vereinigung beider Stoffe vollzog sich in Folge ihrer Vertheilung rasch und zwar ohne Temperaturerhöhung; wohl aber war ein schwaches Sinken um $0,5^{0}$ zu bemerken, obwohl die umgebende Atmosphäre $2^{0}$ wärmer als das Wasser war. Der eben beschriebene, mit den einfachsten Hilfsmitteln ausgeführte Versuch ist zwar nicht geeignet, die thermischen Verhältnisse bei der Bildung des flüssigen Schwefelphosphors mit endgiltiger Schärfe darzulegen; er beweist indess zur Genüge, dass Wärmeentwickelung diesen Vorgang nicht begleitet. Nun spricht im Allgemeinen das Fehlen einer Temperaturerhöhung noch keineswegs gegen die Annahme, dass die betreffende Stoffwandelung ein chemischer Vorgang sei. Wohl aber ist für den vorliegenden Fall eine solche Schlussfolgerung gerechtfertigt, vorausgesetzt, dass der von Thomsen ${ }^{1}$ ) an einigen Beispielen begründete Satz, nach welchem die Wärmeentwickelung der chemischen Processe als Multipla gemeinschaftlicher Constanten auftritt und bei denselben Componenten mit jedem Atom um diese Constante steigt, die vermuthete allgemeine Giltigkeit hat. Die Bildung der Schwefelphosphorverbindungen $\mathrm{P}_{4} \mathrm{~S}_{3}, \mathrm{P}_{2} \mathrm{~S}_{3}$ und $\mathrm{P}_{2} \mathrm{~S}_{5}$ ist bei Anwendung weissen Phosphors von überaus heftigen Reactionen und sehr starken Wärmeentwicklungen begleitet. Leider sind diese Bildungswärmen noch nicht ermittelt; sie würden an der Hand von Thomsen's Satz mit einiger Wahrscheinlich-

1) Dies. Journ. [2] 5, 248. 
keit die Berechnung der den chemischen Verbindungen $P_{4} S$ und $\mathrm{P}_{8} \mathrm{~S}$ entsprechenden Bildungswärmen gestatten. Dass diese aber nicht Null oder negativ sein würden, lässt sich auch ohne jene Zahlen a priori behaupten, da in der Reihe der Phosphorsulfide $\mathrm{P}_{4} \mathrm{~S}_{3}$ der flüssigen Verbindung $\mathrm{P}_{4} \mathrm{~S}$ in Zusammensetzung und Bildungswärme näher stehen würde, als $\mathrm{P}_{2} \mathrm{~S}_{5}$, welches ebenso wie $\mathrm{P}_{4} \mathrm{~S}_{3}$ unter starker Wärmeentwicklung aus den Componenten entsteht. Da aber die Bildung des flüssigen Schwefelphosphors von keiner Temperaturerhöhung begleitet wird, so ist es - die Richtigkeit des Thomsen'schen Satzes vorausgesetzt - sehr unwahrscheinlich, dass er chemischen Kräften seine Entstehung verdankt.

\section{Verhalten beim Abkühlen.}

Berzelius' Phosphorsulfuret „erstarrt nicht bei $0^{0}$, aber einige Grade darunter nimmt es feste Form an und bildet dann ein Gewebe von weissen, durchsichtigen, mikroskopischen Krystallen, die wieder schmelzen, wenn man sie in einer Temperatur von $0^{0}$ oder einigen Graden darüber verweilen lässt." Ich kann diese für die chemische Individualität des Körpers sprechenden Angaben nicht hestätigen. Der meinen eigenen Beobachtungen zu Grunde liegende flüssige Schwefelphosphor war in der oben beschriebenen Weise genau in den der Formel $\mathbf{P}_{4} \mathrm{~S}$ entsprechenden Verhältnissen dargestellt und frei von ungelösten Theilen. Es sei hier bemerkt, dass beim Zusammenfliessen von Phosphor und Schwefel in diesem Mengenverhältnisse noch geringe Antheile Schwefel frei zu sein pflegen, wenn der Phosphor bereits völlig hinweggeschmolzen ist. Doch lösen sich auch diese bald völlig auf, und die erhaltene Flüssigkeit vermag bei gewöhnlicher Temperatur eben so wohl Phosphor, als auch (n geringer Menge) Schwefel aufzunehmen. Der genau nach der Formel $\mathrm{P}_{4} \mathrm{~S}$ zusammengesetzte flüssige Schwefelphosphor verhält sich nun beim Abkühlen keineswegs wie eine einheitliche chemische Verbindung. Er erstarrt nicht bei einer bestimmten Temperatur zu einer homogenen Masse, sondern scheidet schon bei geringer Temperaturerniedrigung auf $8^{0}$ bis $9^{0}$ Phosphorkrystalle $a b$, welche sich theils lose in der resti- 
renden Flüssigkeit befinden, theils an den Wandungen festsitzen, und namentlich dann, wenn sie bei verticaler Stellung der Röhre entstanden sind, die Rhombendodekaëdergestalt gut erkennen lassen. Bei $15^{0}$ löst sich der Phosphor rasch wieder auf, um bei niederen Temperaturen von Neuem auszukrystallisiren. Mehrere, stets unter Ausschluss von Wasser dargestellte Präparate haben mir diese Thatsachen wiederholt bestätigt. Zudem wurden analytische Bestimmungen des ausgeschiedenen festen, sowie des flüssig gebliebenen Theiles ausgeführt, deren Resultate jeden weiteren $Z_{w}$ eifel ausschliessen.

Die ursprüngliche Flüssigkeit enthielt $79,49 \mathrm{pCt}$. P und $20,51 \mathrm{pCt} . \mathrm{S}$ (gemäss der Formel $\mathrm{P}_{4} \mathrm{~S}$ ).

Der bei $9^{0}$ auskrystallisirte Theil enthielt $98,01 \mathrm{pCt}$. $\mathrm{P}$ u. 1,81 pCt. S.

Der bei $9^{0}$ flüssig gebliebene Theil enthielt 75,03 pCt. P u. 24,12 pCt. S.

Der ausgeschiedene Phosphor war offenbar mit Mutterlauge beladen, ron der er sich übrigens auf keine Weise trennen liess.

Während aus $\mathrm{P}_{4} \mathrm{~S}$ beim Abkühleu Phosphor krystallisirt, scheiden sich aus dem zweiten, von Berzelius als chemische Verbindung von der Formel $\mathrm{P}_{2} \mathrm{~S}$ beschriebenen Sulfide (zu dessen Bereitung gelindes Erwärmen nöthig ist, wenn nicht viel Schwefel ungelöst bleiben soll) beim Erkalten Schwefelkrystalle aus. Dieselben enthalten geringe Mengen Phosphor, sind aber durch Umkrystallisiren aus Schwefelkohlenstoff leicht rein zu erhalten. Die Analyse des nicht erstarrten Antheiles ergab 77,87 pCt. P und 22,50 pCt. S.

Auch in Bezug auf das unterphosphorige Sulfid $\mathrm{P}_{2} \mathrm{~S}$ bin ich also gezwungen, Berzelius' Angaben zu widersprechen, nach denen diese Verbindung in der Temperatur, bei welcher $\mathrm{P}_{4} \mathrm{~S}$ erstarre, flüssig bleibe und erst in einer Mischung von Kochsalz und Schnee in mikroskopische Krystalle sich verwandle. Wicke ${ }^{1}$ ) hatte gleichfalls gefunden, dass ein durch Aufösen von Schwefel in $\mathrm{P}_{4} \mathrm{~S}$ erzeugtes Sulfid bei längerem Verweilen an einem kühlen Orte Schwefel-

1) Ann. Chem. Pharm. 86, 115. 
krystalle absetze. Ich kann dem beifügen, dass die offenbar von der Temperatur abhängige Zusammensetzung der restirenden Flüssigkeit bei gewöhnlicher Temperatur der Formel $\mathrm{P}_{4} \mathrm{~S}$ nicht genügend entspricht, und dass Wicke's Angabe, es hinterbleibe stets $\mathrm{P}_{4} \mathrm{~S}$, nur ungefähr richtig ist.

Die Berzelius'schen Schwefelphosphore verhalten sich nach alledem nicht wie chemische Verbindungen. Gegen die etwa noch offenstehende Annahme, dass eine zwischen $\mathrm{P}_{4} \mathrm{~S}$ und $\mathrm{P}_{2} \mathrm{~S}$ stehende flüssige Schwefelphosphorverbindung als chemisches Individuum existire, spricht entschieden die Thatsache, dass aus Phosphor und Schwefel auch bei Gegenwart von übersehüssigem Schwefel bei gewöhnlicher Temperatur Körper entstehen, welche schon bei geringer Abkühlung Phosphor ausscheiden. Unter solchen Umständen kann die Verflüssigung beider Elemente unmöglich das Resultat chemischer Attractionen sein.

\section{Verhalten beim Erhitzen.}

Dagegen sprechen andere Angaben, welche aus Berzelius' Abhandlung in alle Lehrbücher übergegangen und nie bezweifelt worden sind, sehr bestimmt für die Existenz wirklicher chemischer Verbindungen von den Formeln $\mathrm{P}_{4} \mathrm{~S}$ und $\mathrm{P}_{2} \mathrm{~S}$. Von ersterer wird berichtet, dass sie sich in einer Atmosphäre von Wasserstoffgas unverändert überdestilliren lasse, von letzterer, dass sie in sauerstofffreier Atmosphäre bei einer niedrigeren Temperatur, wie der Schwefel, in's Sieden komme, dass sie aber einen höheren Siedepunkt als der Phosphor habe. Allein auch diesen Angaben muss ich auf Grund meiner Erfahrungen widersprechen. Weder für die eine, noch die andere "Verbindung" ist der Siedepunkt mit Bestimmtheit angegeben. Ich prüfte darum zunächst das Verhalten der bei gelinder Wärme nach den Formeln $\mathrm{P}_{4} \mathrm{~S}$ und $\mathrm{P}_{2} \mathrm{~S}$ dargestellten völlig flüssigen Körper bei allmählich gesteigerten Temperaturen in einem indifferenten Gasstrome, und zwar zunächst in Wasserstoffgas. Schon bei gewöhnlicher Temperatur besitzt das mit den beiden Sulfiden in Berührung gewesene, entweichende Gas starken Phosphorgeruch, und bei der Siedetemperatur des Wassers 
entführt dasselbe Phosphor in merkbaren Mengen, welcher sich an den kühlen Stellen des Gefässes (ich benutzte hierbei einen mit doppelt durchbohrtem Korke verschlossenen Probircylinder) als feiner Tröpfchenbeschlag condensirte. Eine Temperatur von $150^{\circ}$ genügte, um bei raschem Gasstrome schon nach kurzer Zeit grosse, zeitweilig zurückfliessende Tropfen entstehen zu lassen, die beim Abkühlen fest wurden und sich bei der Untersuchung als schwefelfrei erwiesen. Der entweichende Wasserstoff war bei Anwendung dieser Temperatur mit Phosphorwasserstoffgas beladen, wie schon der Geruch verrieth; zudem entzündete er sich auch nach völliger Abkühlung beim Austreten an die atmosphärische Luft. Abgesehen hiervon waren nach Ersetzung des Wasserstoffs durch Kohlensäure die Erscheinungen ganz die oben beschriebenen. Der indifferente Gasstrom bringt also schon bei Temperaturen, welche weit unter dem Siedepunkte des Phosphors liegen, diesen zum allmählichei Verdampfen - ein Verhalten, das für das freie Element nichts Auffälliges hat, mit der Annahme einer chemischen Bindung des Phosphors jedoch unvereinbar ist.

Es gelang mir nicht, in beschriebener Weise eine vollständige Zerlegung der flüssigen Schwefelphosphore zu erreichen, weil früher oder später, und einige Male schon bei $100^{\circ}$, innerhalb der Flüssigkeiten eine Reaction eintrat, die oft nur in heftigem Emporwerfen der Masse innerhalb des Gefässes, meist aber in zertrümmernder Explosion sich äusserte, und die sich namentlich dann in heftiger und gefahrbringender Weise vollzog, wenn sie erst bei höheren Temperaturen erfolgte. Sie ist es, welche die Angaben einiger Lehrbücher verschuldet, dass die Bildung des flüssigen Schwefelphosphors unter Umständeu sich unter heftiger, explosionsartiger Reaction vollziehe; so berichtet Bü chner's bekanntes Lehrbuch: "Phosphorsulfuret $\mathrm{P}_{4} \mathrm{~S}$ entsteht unter heftiger Wärmeentwicklung beim Zusammenreiben der Bestandtheile unter Wasser." Der Umstand aber, dass sich diese Reaction auch in den fertig gebildeten, völlig flüssigen Sulfiden $\mathrm{P}_{4} \mathrm{~S}$ und $\mathrm{P}_{2} \mathrm{~S}$ durch Erhitzen hervorrufen lässt, lehrt am besten, dass sie mit der Bildung der Flüssigkeiten selbst Nichts zu 
thun hat, sondern dass ihre Ursache in einer bisher noch nicht zur Geltung gelangten theilweisen chemischen Vereinigung der Componenten zu suchen ist. Es wird durch diese chemische Reaction ein Phosphorsulfid gebildet, welches mit dem auch bei Anwendung von $\mathrm{P}_{2} \mathrm{~S}$ überschüssig bleibenden Phosphor ein flüssiges Gemisch zu bilden scheint. Das Verhalten der "explodirten flüssigen Phosphorsulfide" beim Erkalten wird sehr wahrscheinlich die theilweise erfolgte Vereinigung des Phosphors mit dem Schwefel erkennen lassen; ich bin indess nicht in der Lage, hierüber berichten zu können, da die zur Erlangung genügender Mengen angestellten Versuche an der. Heftigkeit der Explosion scheiterten, die den Arbeitenden mit schweren Verbrennungen bedroht.

Indess gelang es einige Male, durch sehr allmähliches Erhitzen geringer Mengen flüssigen Schwefelphosphors im Paraffinbade nach minder heftiger Reaction Phosphor noch weit unter seinem Siedepunkte mittelst des Kohlensäurestroms zu verflüchtigen und einen Rückstand von festem Schwefelphosphor zu erhalten. Der eine dieser Versuche war bei $150^{\circ}$ so lange fortgesetzt worden, bis keine Phosphordämpfe mehr entwichen. Die Kugelröhre, in welcher operirt wurde, enthielt dann eine durchscheinend gelbe, amorphe Masse, die etwas härter als Paraffin war und sich bei der Analyse als aus

$$
50,96 \text { pCt. P und } 48,23 \text { pCt. } \mathrm{S}
$$

zusammengesetzt erwies. Da die Formel $\mathrm{P}_{4} \mathrm{~S}_{3} 56,4$ pCt. $\mathrm{P}$ verlangt, so schien das Product ein Gemenge von $\mathrm{P}_{4} \mathrm{~S}_{3}$ mit geringen Antheilen eines höheren Sulfids zu sein. Es zeigte das eigenthümliche Verhalten, mit Phosphor bei gewöhnlicher Temperatur, gerade wie der lösliche Schwefel, zu zerfliessen, doch war die Flüssigkeit stark getrübt. Zwei andere Versuche lieferten gleichfalls gelbe amorphe Rückstände, daneben jedoch, und zwar in den engen Theilen der Kugelröhren, geringe Mengen fast weisser nadliger Kryställchen. Diese letzteren werden beim Erhitzen orangegelb, schmelzen dann und verdampfen unter Bildung eines weissen Sublimates. Die mit $76 \mathrm{Mgrm}$. ausgeführte Aualyse derselben ergab:

$$
57,08 \text { pCt. } P \text { und } 41,88 \text { pCt. s. }
$$


Die Verbindung $\mathrm{P}_{2} \mathrm{~S}_{3}$ enthält jedoch 60,76 pCt. $\mathrm{P}$ und 39,24 pCt. S; ich muss darum unentschieden lassen, ob bei der im flüssigen Schwefelphosphor sich vollziehenden Reaction auch $\mathrm{P}_{2} \mathrm{~S}_{3}$ gebildet wird. Dagegen gab die Analyse des einen der gelben Rückstände Zahlen, die der Formel $\mathrm{P}_{4} \mathrm{~S}_{3}$ gut entsprechen, nämlich:

55,88 pCt. P und 44,07 pCt. S.

Die Substanz löste sich leicht und vollständig in Schwefelkohlenstoff und blieb beim Verdunsten des Lösungsmittels auf dem Uhrglase in flach aufliegenden Krystallisationen zurück. Nach alledem scheint beim Erhitzen des flüssigen Schwefelphosphors vorzugsweise $\mathrm{P}_{4} \mathrm{~S}_{3}$ gebildet zu werden.

Im Anschluss an diese Versuche sei eine Erscheinung erwähnt, die beim Erhitzen der flüssigen Sulfide im indifferenten Gasstrome stets zu beobachten war. An der Austrittsstelle desselben zeigt sich nämlich ein gelbes Phosphorflämmchen, in welchem die weggeführten Dämpfe unter Entwicklung eines leichten weissen Rauches verbrennen. Dabei ist die Wärmetönung dieses Flämmchens eine überraschend schwache, so dass es Papier nicht zu bräunen vermag und selbst der Hand nur wenig fühlbar ist. Die starke Verdünnung der Phosphordämpfe mag daran ebenso Schuld sein wie der Umstand, dass bei der Verbrennung nur phosphorige Säure entsteht (beim Verbrennen zu $\mathrm{P}_{2} \mathrm{O}_{5}$ wird die fünffache Wärmemenge erzeugt). Die phosphorige Säure wurde durch Absaugen des weissen Rauches mittelst eines über die Flamme gestülpten Trichters und Durchleiten desselben durch Silberlösung nachgewiesen, wobei binnen Kurzem ein reichlicher Niederschlag von reducirtem Silber entstand. Es bedarf wohl kaum der Erwähnung, dass diese interessante Erscheinung des "kalten Phosphorflämmchens" sich ebenso sicher und zugleich bequemer durch Anwendung blossen Phosphors hervorrufen lässt.

\section{Verhalten gegen Lösungsmittel.}

Im Eingange dieser Abhandlung citirte ich zwei in Gmelin-Kraut's Handbuche zu findende Angaben über das Verhalten des flüssigen Schwefelphosphors gegen Lösungs- 
mittel überhaupt und gegen Schwefelkohlenstoff insbesondere. Ich muss indess die Behauptung, dass letzterer vorzugsweise den Phosphor ansziehe und den meisten Schwefel in Verbindung mit etwas Phosphor abscheide, als unrichtig zurückweisen. Schwefelkohlenstoff mischt sich mit flisssigem Schwefelphosphor in jedem Verhältniss und verhindert schon in geringen Mengen die Ausscheidung fester Substanzen beim Abkühlen. Allerdings löst Schwefelkohlenstoff den Phosphor reichlicher als den Schwefel, aber im vorliegenden Falle wirkt dem Bestreben des Schwefels, bei niederer Temperatur auszukrystallisiren, die Attraction des Phosphors entgegen; alle drei Bestandtheile der Mischung sind durch gegenseitige Anziehungen an einander gekettet. Wir kennen keine Flüssigkeit, die nur einen der beiden Bestandtheile löste; darum vermischen sich gleich dem Schwefelkoblenstoff alle Lösungsmittel, die etwa in Vorschlag kommen könnten, mit dem flüssigen Schwefelphosphor, ohne eines der Elemente abzuscheiden.

Indess gelingt die Zerlegung wenigstens theilweise mit Hilfe eines complicirteren Verfahrens, dessen Darlegung einige Vorbemerkungen erfordert. Schüttelt man ęine concentrirte Auflösung von Schwefel in Schwefelkohlenstoff mit Alkohol, Aether, Chloroform, Petroleumäther oder einer anderen mit Schwefelkohlenstoff mischbaren Flüssigkeit, die nur wenig Lösungsvermögen für Schwefel besitzt, so wird der Schwefel zum grössten Theile ansgeschieden, und über ihm befindet sich dann das Flüssigkeitsgemisch, welches je nach der Menge des vorhandenen Schwefelkohlenstoffs grössere oder geringere Mengen Schwefel in Lösung hält. Geringe Mengen dieser Lösungsmittel gehen auch ohne Schütteln, gleichfalls unter Schwefelabscheidung, rasch in die Schwefel-Schwefelkohlenstofflösung über; so wird bei Zusatz von wenig Chloroform Schwefel in flachen Krystallen niedergeschlagen, die stetig wachsend zu Boden sinken - eine Erscheinung, die mit dem Auskrystallisiren des chlorsauren Kalis beim Erkalten seiner heiss gesättigten Lösung einige Aehnlichkeit hat. Auch Petroleumäther zerlegt die SchwefelSchwefelkohlenstofflösung in interessanter Weise; schichtet 
man nämlich jenen vorsichtig über die Schwefellösung, so sinken von der Trennungsfläche beider Flüssigkeiten in dem Maasse, als die Vereinigung des Petroleumäthers mit dem Schwefelkohlenstoff fortschreitet, nadelige Krystalle von Schwefel stetig nieder. Diese Erscheinungen sind wohl kaum ganz neu; ich glaubte aber, auf sie besonders aufmerksam machen zu dürfen, weil sie für experimentelle Vorführung sehr geeignet und vielleicht auch für die Krystallographie des Schwefels von Bedeutung sind.

Phosphor wird aus seiner Auflösung in Schwefelkohlenstoff durch die genannten Flüssigkeiten in Tröpfchen ausgefällt, die neben Phosphor wechselnde Mengen von Schwefelkohlenstoff enthalten; nach Wiederholung des Verfahrens wird auch dieser Rest aufgenommen und Phosphor in fester Gestalt abgeschieden.

Aus einer Lösung von Phosphor und Schwefel in Schwefelkohlenstoff gelangt nur dann im Anfang reiner Schwefel zur Ausscheidung, wenn er in bedeutendem Ueberschusse vorhanden ist. Andernfalls sondert sich eine schwefelkohlenstoffärmere Phosphor-Schwefel-Schwefelkohlenstofflösung ab, die nach gänzlicher Entfernung des Schwefelkohlenstoffes durch Ausschütteln mit dem Fällungsmittel flüssigen Schwefelphosphor zurücklässt, der je nach den verwendeten Phosphor- und Schwefelmengen verschieden zusammengesetzt ist. Der flüssige Schwefelphosphor selbst nun, der für diese Versuche nach den der Formel $\mathrm{P}_{4} \mathrm{~S}$ entsprechenden Verhältnissen dargestellt wurde, lässt sich durch abwechselndes Behandeln mit Schwefelkohlenstoff und einem minder guten Lösungsmittel, z. B. Chloroform, theilweise zerlegen, wie folgender Versuch beweist.

Das Sulfid wurde mit dem gleichen Volum Schwefelkohlenstoff versetzt und dann mit $2 \mathrm{Vol}$. Chloroform geschüttelt. Dabei schied sich unter dem Schwefelkohlenstoff-Chloroform-Gemisch scheinbar unveränderter Schwefelphosphor von wenig vermindertem Volumen ab, welcher jedoch ca. 1/8 Vol. Schwefelkohlenstoff enthielt; auch dieser Rest wurde durch Schütteln mit 1 Vol. Chloroform fast ganz entfernt. Das ganze Verfahren wurde ein zweites und drittes Mal wieder- 
holt, wobei das Sulfid breiig wurde. Die vierte Wiederholung aber ergab festen Phosphor, und zwar dem Volumen nach ungefähr die Hälfte des angewendeten Schwefelphosphors. Die Analyse desselben ergab

$$
98,53 \text { pCt. P und } 0,74 \text { pCt. S. }
$$

Ich lasse es bei diesem einen Boispiele bewenden. Von den vielen Methoden, die zur Zerlegung des flüssigen Schwefelphosphors mit Hilfe von Lösungsmitteln versucht wurden, ergab die beschriebene immer noch das beste Resultat, so sehr auch dieses selbst zu wünschen übrig lässt.

Phosphor und Polysulfide.

Bei unparteiischer Behandlung der Frage nach der Natur der flüssigen Phosphorsulfide dürfen einige Thatsachen nicht übersehen werden, die auf den ersten Blick geeignet sind, gegen das durch alle bisherigen Versuche gewonnene Resultat, also für die chemische Individualität dieser Körper zu sprechen. Die erste dieser Thatsachen ist die Entstehung des flüssigen Schwefelphosphors aus Mehrfach-Schwefelkalium und Phosphor, deren Kenntniss wir R. Böttger ${ }^{1}$ ) verdanken. Dieser sättigte eine alkoholische Schwefelkaliumlösung mit Schwefel, erhitzte darin wohl gereinigten Phosphor zum Schmelzen, schüttelte in einem Arzneiglase tüchtig durch und erhielt nach viertägigem Stehen am dunklen Orte fast wasserhellen Schwefelphosphor. Ich kann dem beifügen, dass auch wässrige Lösungen von Kalium - und Ammoniumpolysulfid an schmelzenden Phosphor Schwefel abgeben, und dass nach einigen Tagen (die Zeit ist von der Temperatur und Concentration der Lösung abhängig) der Phosphor ein Maximum von Schwefel aufgenommen hat, welches, wie folgende Zahlen beweisen, dem der Formel $\mathrm{P}_{4} \mathrm{~S}$ entsprechenden Schwefelgehalte von 20,52 pCt. nur nahe kommt, nicht aber gleich ist:

1) Dies. Journ. 12, 358 . 
Flüssiger Schwefelphosphor aus Phosphor und

1. alkoholischem Kaliumpolysulfid enthielt nach 3 Tagen 18,50 pCt., nach 6 Tagen 19,37 pCt. $\mathrm{s}$,

2. wässrigem Kaliumpolysulfid enthielt nach 3 Tagen $19,08 \mathrm{pCt}$., nach 6 Tagen 23,57 pCt. $S$,

3. wässrigem Ammoniumpolysulfid enthielt nach 3 Tagen 22,14 pCt., nach 6 Tagen $24,75 \mathrm{pCt}$. $\mathrm{S}$.

Dabei ist zu bemerken, dass diese Flüssigkeiten täglich ca. 8 Stunden bis zum Schmelzen des Phosphors erhitzt und öfters durchgeschüttelt wurden. Die auf diese Weise erhaltenen flüssigen Schwefelphosphore verhalten sich ganz wie die unmittelbar aus Phosphor und Schwefel dargestellten. Sie geben beim Erhitzen im indifferenten Gasstrome Phosphor ab und liefern beim Abkühlen Phosphorkrystalle.

Nach den Versuchen Böttg er's, welcher nach dem Schütteln das Gefäss bei Sauerstoffzutritt offen stehen liess, muss es ungewiss bleiben, ob auch Phosphor allein dem Polysulfid Schwefel entzieht. Meine Versuche ergeben dies mit Bestimmtheit, da sich bei ihnen die Bildung des Schwefelphosphors in Flaschen vollzog, die fast ganz mit Flüssigkeit gefüllt und durch Korke gut verschlossen waren. Die Bildung des flüssigen Schwefelphosphors aus Phosphor und einer schwefelreichen chemischen Verbindung scheint dafür zu sprechen, dass es chemische Attractionen seien, die beide Elemente zusammenführen. Indess sind Thatsachen bekannt, welche zeigen, dass in analoger Weise Verbindungen durch andere Stoffe zerlegt werden können, ohne dass diese eine chemische Attraction äussern. So verlieren viele krystallwasserhaltige Salze an trockner Luft das Krystallwasser mehr oder minder vollständig, und die zerlegende Kraft ist lediglich in der molecularen Anziehung zu suchen, welche die Luft gegen Wasser äussert. Will man indess die Analogie solcher wasserhaltiger Verbindungen, die ja keine eigentlichen Atomverbindungen sind, mit den Polysulfiden verneinen, so dürfte die Beobachtung Schöne's ${ }^{1}$ ), dass Alkohol aus einer concentrirten wässrigen Lösung von Kaliumpenta-

เ) Gmelin-Kraut 2, 38. 
sultid wasserhaltiges Tetrasulfid fällt, indem er Schwefel (und Polysulfid) auflöst, wohl den sichersten Beweis liefern, dass Mehrfach-Schwefelkalium durch Körper zerlegt werden kann, welche dabei nur als Lösungsmittel wirken.

Wie ich gefunden habe, entsteht auch aus Wasserstoffsupersulfid und Phosphor flüssiger Schwefelphosphor. Zunächst vereinigt sich der Phosphor mit dem darin gelösten überschüssigen Schwefel, und erst dann vollzieht sich die Zersetzung des Sulfides selbst, wie dies die eintretende Schwefelwasserstoffentwicklung kundgiebt. Bei $15^{0}$ ist die Gasentwicklung spärlich, bei $35^{\circ}$ aber lebhaft, während reines Supersulfid allein bei dieser Temperatur nur langsam zerfällt. Schwefelphosphor ist übrigens in jedem Verhältniss mit Wasserstoffsupersulfid mischbar. Die Zerlegung des unbeständigen Wasserstoffsupersulfids durch Phosphor hat weniger Auffallendes als die des Kaliumpolysulfids. Doch lässt sich auch diese, wie gezeigt wurde, mit der Auffassung der flüssigen Phosphorsulfide als Gemisch recht wohl in Einklang bringen. Die Aufnahme ungefähr gleicher, etwa der Formel $\mathrm{P}_{4} \mathrm{~S}$ entsprechender Schwefelmengen aber bedeutet nichts Anderes, als dass das gegenseitige Lösungsbestreben beider Componenten für gleiche Temperaturen dasselbe ist. Dass beide bei verschiedenen Temperaturen in verschiedenem Grade fähig sind, einander in Lösung zu halten, geht aus dem früher über das Verhalten der "Verbindungen" $\mathrm{P}_{4} \mathrm{~S}$ und $\mathrm{P}_{2} \mathrm{~S}$ beim Abkühlen Gesagten zur Genüge hervor.

Die Zersetzung, welche die flüssigen Schwefelphosphore durch Wasser und namentlich durch Wasserdampf erleiden, ist die zweite, die chemische Individualität dieser Körper nur scheinbar stützende Thatsache. Weder Schwefel noch Phosphor zerlegen für sich allein das Wasser, und es erscheint auf den ersten Blick auffallend, dass ein blosses Gemisch beider zu solcher Zersetzung fähig sein sollte. Es giebt indess mehrere Beispiele, welche zeigen, dass zwei nur gemeinsam auf einen dritten einwirkende Stoffe dies keineswegs nur dann $\mathrm{zu}$ thun vermögen, wenn sie chemisch verbunden sind. Die Anführung der bekannten Reaction: 


$$
3 \mathrm{C}+6 \mathrm{Cl}+\mathrm{Al}_{2} \mathrm{O}_{3}=\mathrm{Al}_{2} \mathrm{Cl}_{6}+3 \mathrm{CO}
$$

wird auch ohne weitere Darlegung das Gesagte bestätigen.

Die Zersetzung des flüssigen Schwefelphosphors durch Wasser spricht also mit ebenso wenig Bestimmtheit für deren chemische Individualität, wie ihre Entstehung aus Alkalipolysulfid und Phosphor.

Wenn nun aber die flüssigen Phosphorsulfide keine chemischen Verbindungen sind, zu welcher Klasse von Stoffaggregationen gehören sie dam? Wie in den Auflösungen in Schwefelkohlenstoff erreichen Schwefel und Phosphor in diesen Körpern die Grenze ihrer physischen Theilbarkeit, und wie bei Lösungsvorgängen nur einer von zwei Stoffen den festen Aggregatzustand einbüsst, da der andere bereits flüssig ist, so sind es hier beide Componenten, welche durch eine gegenseitige Anziehung ihrer Moleküle aus dem festen in den flüssigen Zustand übergehen. Eben so wenig ein mechanisches Gemisch wie eine chemische Verbindung reiht sich der flüssige Schwefelphosphor zur grossen Zahl der mannigfaltigen Stoffgruppirungen, die ihre Entstehung molecularen Kräften verdanken, ohne dass sie Molekülverbindungen im Sinne der modernen Chemie wären. ${ }^{1}$ ) Unter diesen Stoffgruppirungen, denen C. Jakob ein aufklärendes Schriftchen ${ }^{2}$ ) gewidmet hat, und für die vielleicht im Gegensatz zu den Molekülverbindungen und den mechanischen Gemengen der Ausdruck "mechanische Verbindungen" passend ist, stehen die Lösungen dem flüssigen Schwefelphosphor am nächsten. Kennen wir doch auch Fälle, dass Lösungen unmittelbar aus festen Componenten hervorgehen. Die Verflüssigung fester, in Wasser löslicher Salze in Berührung mit Eis bietet eine vollkommene Analogie zur Bildung des flüssigen Schwefelphosphors aus seinen Elementen.

Freiberg i. S., Laboratorium der Bergakademie.

1) A. Naumann, Allg. u. phys. Chem., S. 80.

$\left.{ }^{2}\right)$ Dr. C. Jakob, Molekulanz. u. Molekülverb. Stuttgart. 